Provided for non-commercial research and education use. Not for reproduction, distribution or commercial use.

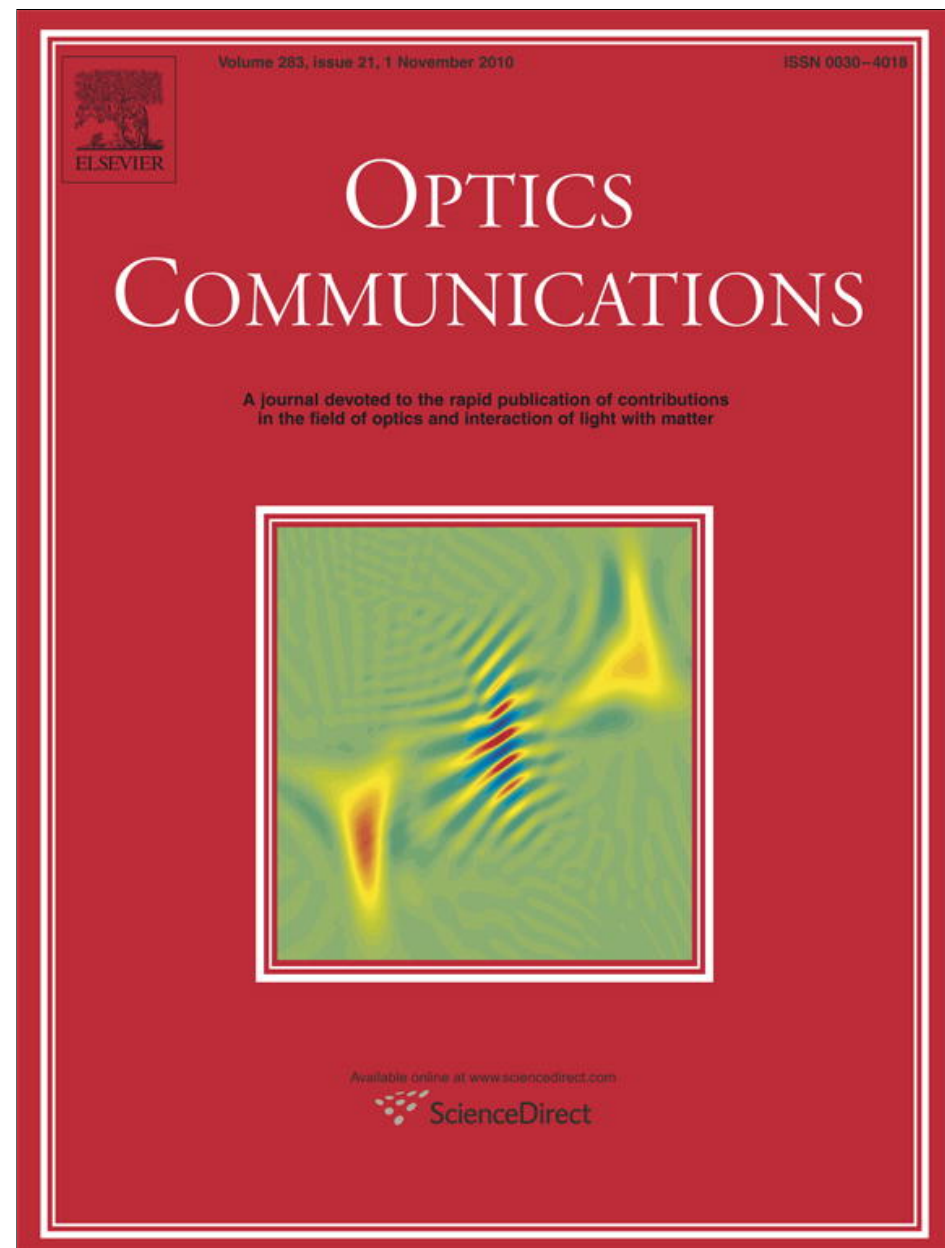

This article appeared in a journal published by Elsevier. The attached copy is furnished to the author for internal non-commercial research and education use, including for instruction at the authors institution and sharing with colleagues.

Other uses, including reproduction and distribution, or selling or licensing copies, or posting to personal, institutional or third party websites are prohibited.

In most cases authors are permitted to post their version of the article (e.g. in Word or Tex form) to their personal website or institutional repository. Authors requiring further information regarding Elsevier's archiving and manuscript policies are encouraged to visit:

http://www.elsevier.com/copyright 


\title{
Off-resonant activation of optical emission
}

\author{
David L. Andrews *, David S. Bradshaw \\ School of Chemistry, University of East Anglia, Norwich NR4 7TJ, United Kingdom
}

\section{A R T I C L E I N F O}

\section{Article history:}

Received 13 May 2010

Received in revised form 14 June 2010

Accepted 14 June 2010

\section{Keywords:}

Nonlinear optics

Laser emission

Enhanced fluorescence

Optical transistor

\begin{abstract}
A B S T R A C T
Recent reports have identified a three-wave optically parametric mechanism for the active enhancement of fluorescence using off-resonant radiation. In this Letter it is shown by numerical simulation that the output of a laser system optically pumped just below threshold, can be strongly enhanced by this mechanism, using an ancillary beam of moderate intensity. The electrodynamics and kinetics of the nonlinear optical mechanism are analyzed, model calculations performed, and the output is illustrated graphically. The response demonstrates a novel method for achieving all-optical transistor action.
\end{abstract}

(C) 2010 Elsevier B.V. All rights reserved.

\section{Introduction}

In the sphere of parametric nonlinear optics, a facility to achieve switching and coherent frequency conversion is well known and its mechanisms are widely employed. The high efficiency of such processes are largely due to the coherence of the fundamental interactions, sustained by wave-vector matching and compounded by the development of stimulated emission in the output. Recently it has emerged that the basic process of optical emission can itself engage with off-resonant laser light, the strength of this mechanism owing to the stimulated forward scattering character of the coupling. In this Letter it is shown that the output of a laser system optically pumped just below threshold can be strongly enhanced by this mechanism, given the throughput of an ancillary beam with moderate intensity. Part of the interest in such a scheme lies in its possible relevance to all-optical transistor action which, like its electronic antecedent, can effect the switching or amplification of a source under the control of a signal input. Several other novel schemes for the realization of this type of action have recently been proposed, including one based on saturated absorption [1], electromagnetic induced transparency (EIT) [2-5], the optical Kerr effect [6-8], nonlinear transmission through coupling with surface plasmons [9-11], and beam filament rotation by application of a signal beam $[12,13]$.

It can be anticipated that the desired characteristics of any practicable all-optical transistor will depend crucially on the engagement of a strongly nonlinear optical response. The scheme proposed in the present work is based on a third-order nonlinearity - its effect enhanced by stimulated emission - operating within a system designed to exploit the highly nonlinear response observed at the threshold for laser emission. In

\footnotetext{
* Corresponding author.

E-mail addresses: david.andrews@physics.org, D.L.Andrews@uea.ac.uk (D.L. Andrews).
}

contrast to the work of Hwang et al. [1], stimulated emission here primarily occurs in the course of forward scattering by a throughput beam whose optical frequency is purposely off-set from resonance. Detailed analysis shows that this beam, acting as the input signal, modifies the kinetics of emission and can thereby lead to an enhanced output $[14,15]$. The results of calculations for three-level laser systems highlight a significant potential for all-optical transistor action.

\section{Method and results}

Consider a typical three-level laser system optically pumped within a microcavity. The kinetics of emission are primarily determined by a pump rate $R_{p}$ driving population from the ground state $E_{0}$ into a metastable upper level $E_{2}$, with stimulated emission as $E_{2}$ decays to $E_{1}$, and ultrafast relaxation from $E_{1}$ (Fig. 1). Following Siegman [16], the standard rate equations for the temporal behaviour of the cavity photon number, $n$, and the $E_{2}$ population, $N_{2}$, are as follows:

$$
\begin{aligned}
& \frac{d n}{d t}=K(n+1) N_{2}-\gamma_{c} n, \\
& \frac{d N_{2}}{d t}=R_{p}-n K N_{2}-\gamma_{2} N_{2} .
\end{aligned}
$$

Here, the population of $E_{1}$ is assumed to be negligible; $K$ denotes the coupling coefficient for the laser transition, and $\gamma_{c}, \gamma_{2}$ signify the cavity and population decay rates, respectively. Under steady-state conditions, Eqs. (1) and (2) give the result;

$$
n=\frac{R_{p}-g \gamma_{c} p+\left[\left(R_{p}-g \gamma_{c} p\right)^{2}+4 \gamma_{c} R_{p}\right]^{\frac{1}{2}}}{2 \gamma_{c}} .
$$




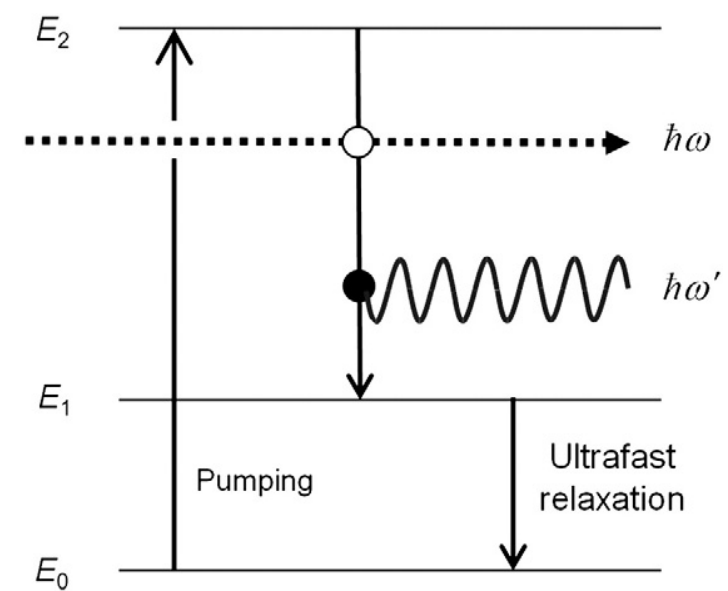

Fig. 1. Energy level diagram of the three-level laser system: black vertical arrows denote electronic transitions, the wavy line represents emission $\left(\hbar \omega^{\prime}\right)$, and the offresonant beam $(\hbar \omega)$ is the dashed arrow. The black and open dots symbolize one and two matter-photon interactions, respectively.

where $\mathrm{g}=\gamma_{2} / \gamma_{\mathrm{rad}}$ and $K=\gamma_{\mathrm{rad}} / p$ (in which $\gamma_{\mathrm{rad}}$ is the radiative decay rate and $p$ is the number of resonant cavity modes). The relaxation from $E_{2}$ into $E_{1}$ is not entirely radiative, i.e. $\gamma_{2} \neq \gamma_{\text {rad, }}$, since nonradiative relaxation processes (lattice phonons, wall collisions etc.) contribute to the decay. For present calculational purposes, given that the level $E_{2}$ decay rate $\gamma_{2}$ subsumes - but is dominated by - the rate of radiative decay, we assume $g=5 / 4$ in the absence of the off-resonant input signal considered below. From Eq. (3), employing typical values $p=10^{10}$ and $\gamma_{c}=10^{8} \mathrm{~s}^{-1}$, the familiar vertical climb in cavity photons at laser threshold emerges, as graphically illustrated by Fig. 2 (solid line).

All-optical control of such a pumped active medium may be achieved by nonlinear optical engagement of the laser emission with stimulated

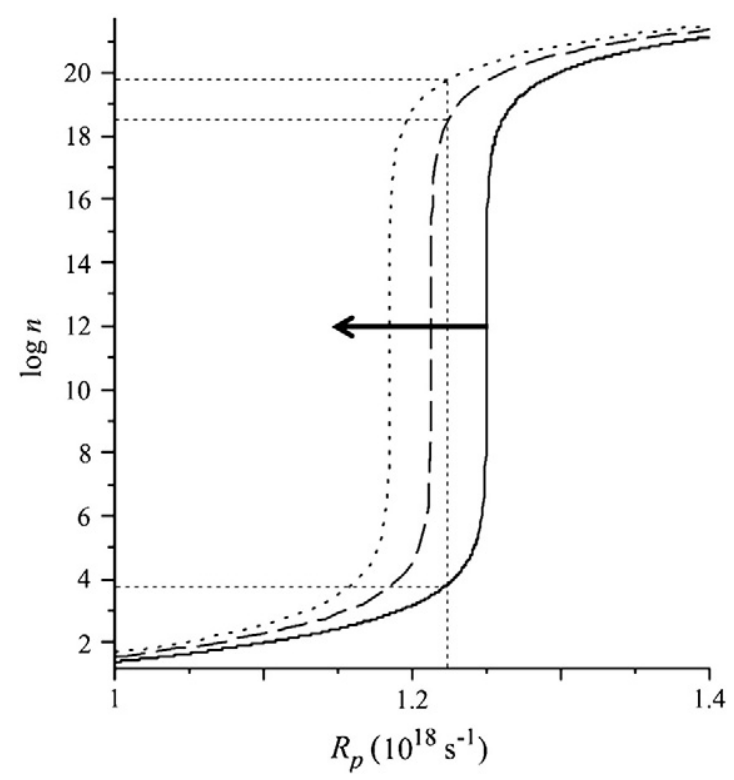

Fig. 2. Plot of $\log n$, where $n$ is the number of cavity photons, against the pumping rate $R_{p}$, for absent (solid line) and present signal beam; example irradiances of the latter are $2 \times 10^{11} \mathrm{~W} \mathrm{~cm}^{-2}$ (dashed) and $4 \times 10^{11} \mathrm{~W} \mathrm{~cm}^{-2}$ (dotted). Horizontal arrow illustrates a movement of the lasing threshold to the left for increasing laser intensities. The vertical dotted line represents a constant $R_{p}$ at which, on introduction of the signal beam, transistor action produces above threshold operation (denoted by the upper pair of horizontal dotted lines). elastic forward scattering of off-resonant (signal) laser pulses, effecting a modification to the dipole transition moment for the $E_{2} \rightarrow E_{1}$ laser transition $[14,15]$. The mechanism fundamentally entails three matterphoton interactions (Fig. 1); photons annihilated and created into the signal radiation mode (which thus emerges unchanged) are coupled with the photon emission. The intensity of emission, $I^{\prime}\left(\Omega^{\prime}\right)$, (or power per unit solid angle) follows from Fermi's Rule [17] multiplied by the emitted photon energy, $\hbar \omega^{\prime}[18]$. Hence, the net intensity is determined from $\mathrm{I}^{\prime}\left(\Omega^{\prime}\right) \mathrm{d} \Omega^{\prime}=2 \pi \rho \omega^{\prime}\left|M^{(1)}+M^{(3)}\right|^{2}$, where $\rho$ is the density of radiation states; $M^{(1)}$ and $M^{(3)}$ are the quantum amplitudes for firstand third-order interaction processes, respectively - the former being the usual result for optical emission, the latter its leading nonlinear correction. The sought optical effects depend on the relative signs of the first- and third-order amplitudes, which are of primarily real character; a common sign will lead to emission enhancement, opposite signs its suppression. To proceed, the following detailed result has been determined in recent work [14];

$$
\begin{aligned}
I^{\prime}\left(\Omega^{\prime}\right)= & \left(\frac{\omega^{\prime 4}}{8 \pi^{2} \varepsilon_{0} c^{3}}\right)\left[e_{i}^{\prime} e_{j}^{\prime} \mu_{i}^{12} \bar{\mu}_{j}^{12}+\left(I / c \varepsilon_{0}\right) e_{i}^{\prime} e_{j} e_{k} e_{l}^{\prime} \chi_{i j k}^{12}\left(\omega^{\prime} ;-\omega, \omega\right) \bar{\mu}_{l}^{12}\right. \\
& \left.+\left(I^{2} / 4 c^{2} \varepsilon_{0}^{2}\right) e_{i}^{\prime} e_{j} e_{k} e_{l}^{\prime} e_{m} e_{n} \chi_{i j k}^{12}\left(\omega^{\prime} ;-\omega, \omega\right) \bar{\chi}_{l m n}^{12}\left(\omega^{\prime} ;-\omega, \omega\right)\right],
\end{aligned}
$$

where successive terms in square brackets originate from $\left|M^{(1)}\right|^{2}$, $M^{(1)} \bar{M}^{(3)}$ (plus its conjugate) and $\left|M^{(3)}\right|^{2}$, respectively. The decay transition dipole moment is designated by the shorthand notation $\boldsymbol{\mu}^{12}=\langle 1|\boldsymbol{\mu}| 2\rangle-$ with $|1\rangle$ and $|2\rangle$ denoting the states of levels $E_{1}$ and $E_{2}$, respectively. Eq. (4) deploys the implied summation convention for repeated (Cartesian) indices, and $I$ is the intensity (irradiance) of the input signal, with $\mathbf{e}^{\prime}$ and $\mathbf{e}$ representing the linear polarization unit vectors of emission and signal photons, respectively.

The key parameter in Eq. (4) is the nonlinear transition (inelastic) tensor, $\chi_{i j k}^{12}\left(\omega^{\prime} ;-\omega, \omega\right)$, mediating radiative decay of the molecular excited state. Unlike a parametric susceptibility, this tensor registers an inelastic (energy loss) process - in consequence of which, the first frequency parameter $\omega^{\prime}$, registering emitter decay, differs from the sum of those which follow in the argument of $\chi$. The explicit form of this tensor is determined from well-attested and reported methods [18-21], and the result is given by:

$$
\begin{aligned}
\chi_{i j k}^{12}\left(\omega^{\prime} ;-\omega, \omega\right)= & \sum_{r} \sum_{s \neq 2}\left(\frac{\mu_{i}^{1 s} \mu_{j}^{s r} \mu_{k}^{r 2}}{E_{s 2}\left(E_{r 2}-\hbar \omega\right)}+\frac{\mu_{i}^{1 s} \mu_{k}^{s} \mu_{j}^{r 2}}{E_{s 2}\left(E_{r 2}+\hbar \omega\right)}\right) \\
& +\sum_{r} \sum_{s}\left(\frac{\mu_{j}^{1 s} \mu_{i}^{s r} \mu_{k}^{r 2}}{\left(E_{s 2}-\hbar \omega+\hbar \omega^{\prime}\right)\left(E_{r 2}-\hbar \omega\right)}+\frac{\mu_{k}^{1 s} \mu_{i}^{s r} \mu_{j}^{r 2}}{\left(E_{s 2}+\hbar \omega+\hbar \omega^{\prime}\right)\left(E_{r 2}+\hbar \omega\right)}\right) \\
& +\sum_{r \neq 1} \sum_{s}\left(\frac{\mu_{j}^{1 s} \mu_{k}^{s r} \mu_{i}^{r 2}}{\left(E_{s 2}-\hbar \omega+\hbar \omega^{\prime}\right)\left(E_{r 2}+\hbar \omega^{\prime}\right)}+\frac{\mu_{k}^{1 s} \mu_{j}^{s r} \mu_{i}^{r 2}}{\left(E_{s 2}+\hbar \omega+\hbar \omega^{\prime 2}\right)\left(E_{r 2}+\hbar \omega^{\prime}\right)}\right),
\end{aligned}
$$

where $\omega$ is the signal beam frequency, and the transition moments are defined in the same manner as $\boldsymbol{\mu}^{12} ; r$ and $s$ are intermediate states, equating to either 0,1 or 2 in the three-level system (except where precluded in certain summations, as indicated above), and $E_{x y}=E_{x}-E_{y}$ is an energy difference between two such states. In passing, we observe that the nonlinear mechanism may alternatively be interpreted in terms of a 'dressing' of the emitter states by the throughput beam, manifest in a modification to the $E_{2} \rightarrow E_{1}$ transition moment; although a different derivation method ensues, the same expression as Eq. (5) will emerge.

Continuing, we may suppose $\omega^{\prime}<\omega$, and that these frequencies determine an offset, $\Delta E=E_{20}-\hbar \omega-\hbar \omega^{\prime}$, a small fraction of the 
energy for a typical electronic transition. Under these conditions the fourth term of Eq. (5) is dominant, delivering the following:

$e_{i}^{\prime} e_{j} e_{k} \chi_{i j k}^{12}\left(\omega^{\prime} ;-\omega, \omega\right) \approx \frac{\mu^{3}}{\Delta E\left(\Delta E+\hbar \omega^{\prime}\right)}$.

Here and henceforth, it is assumed that the relevant transition dipole moment components, now simply represented as $\mu$, have broadly similar magnitudes and direction - in calculations on specific systems, this approximation can of course be surrendered for greater accuracy. It should be observed that both factors in the denominator of Eq. (6) have negative values, so that the resulting tensor is always positive and, thus, denotes enhanced emission; under other conditions the susceptibility components may assume a negative value, representative of reduced emission. On insertion of Eq. (6) into Eq. (4), typical values of $I^{\prime}\left(\Omega^{\prime}\right)$ may now be calculated for various signal beam intensities.

As indicated above, it is evident that the initial and final terms on the right-hand side of Eq. (4) correspond to spontaneous emission and the nonlinear coupling process, respectively. However, it is the second term (linear in I), signifying a quantum interference of these two processes, that represents the leading correction. With this in mind, the degree of enhancement (or in other cases any suppression) of the emission can be measured by taking the ratio of the second term against the first; the corresponding parameter $\eta$ may be approximated as:

$\eta=\frac{I \mu^{2}}{c \varepsilon_{0} \Delta E\left(\Delta E+\hbar \omega^{\prime}\right)}$.

Returning to Eq. (3), it is clear that the variable $g$ will be affected by introduction of the input signal beam, since the radiative decay rate, $\gamma_{\text {rad, }}$, and population decay rate, $\gamma_{2}$, both thereby suffer change (but to differing degrees); the non-radiative decay rate, $\gamma_{n r}$, can be assumed to be constant. By simple manipulation, an expression for $g$ is given by;

$g(I)=1+\frac{1-Y(I)}{Y(I)+\eta Y(I)}$,

where $Y(I)=\gamma_{\mathrm{rad}}(I) / \gamma_{2}(I)$ and $\gamma_{n r} / \gamma_{2}(I)=1-Y(I)$. With the previous condition $g=5 / 4$ for $I=0$, and adopting indicative values $\mu=16 \times 10^{-30} \mathrm{C} \mathrm{m}, \Delta E=10^{-20} \mathrm{~J}$ and $\hbar \omega^{\prime}=10^{-19} \mathrm{~J}$, inserting Eq. (8) into Eq. (3) generates the results illustrated by two further curves on Fig. 2. For a constant pumping rate at a level indicated by the dotted vertical line, the system operates below threshold when no signal laser is present; on introduction of an off-resonant beam with an irradiance approaching $2 \times 10^{11} \mathrm{~W} \mathrm{~cm}^{-2}$, the output climbs by fourteen orders of magnitude, rising to sixteen orders if the signal input is doubled - typical of a laser operating at threshold. Transistor action with respect to the signal beam is clearly exhibited.

\section{Discussion}

The present theoretical analysis, based on off-resonant activation of laser emission, has identified a potentially new basis for achieving all-optical transistor action. The relatively simple optical configuration offers advantages of ultrafast response with high repetition rate, high efficiency, and a straightforward experimental setup. Moreover, the general scheme is based on a fundamental principle that is not limited to operation with any one specific material. In the broad context of research on all-optical methods of switching and amplification, such a scheme now invites experimental exploration with a view to potential applications. The realization of a material system that can prove suitable for implementing this mechanism will require the identification of active media for which the key tensor parameters can be optimized; all the necessary theory is now in place.

\section{Acknowledgement}

The authors thank the Leverhulme Trust for funding this research.

\section{References}

[1] J. Hwang, M. Pototschnig, R. Lettow, G. Zumofen, A. Renn, S. Götzinger, V. Sandoghdar, Nature 460 (2009) 76.

[2] B.S. Ham, P.R. Hemmer, Phys. Rev. Lett. 84 (2000) 4080.

[3] H. Schmidt, R.J. Ram, Appl. Phys. Lett. 76 (2000) 3173.

[4] P. Bermel, A. Rodriguez, S.G. Johnson, J.D. Joannopoulos, M. Soljacic, Phys. Rev. A 74 (2006) 043818

[5] C.H. Raymond Ooi, New J. Phys. 10 (2008) 123024

[6] M.F. Yanik, S.H. Fan, M. Soljacic, J.D. Joannopoulos, Opt. Lett. 28 (2003) 2506.

[7] N. Moll, R. Harbers, R.F. Mahrt, G.L. Bona, Appl. Phys. Lett. 88 (2006) 171104.

[8] M.R. Singh, R.H. Lipson, J. Phys. B 41 (2008) 015401.

[9] C. Janke, J.G. Rivas, P.H. Bolivar, H. Kurz, Opt. Lett. 30 (2005) 2357.

[10] G.A. Wurtz, R. Pollard, A.V. Zayats, Phys. Rev. Lett. 97 (2006) 057402

[11] D.E. Chang, A.S. Sorensen, E.A. Demler, M.D. Lukin, Nat. Phys. 3 (2007) 807

[12] A.M.C. Dawes, L. Illing, S.M. Clark, D.J. Gauthier, Science 308 (2005) 672.

[13] S. Schumacher, N.H. Kwong, R. Binder, A.L. Smirl, Phys. Status Solidi RRL 3 (2009) 10.

[14] D.S. Bradshaw, D.L. Andrews, J. Phys, Chem. A. 113 (2009) 6537.

[15] D.S. Bradshaw, D.L. Andrews, Phys. Rev. A 81 (2010) 013424.

[16] A.E. Siegman, Lasers, Oxford University Press, Oxford, 1986, p. 510.

[17] L. Mandel, E. Wolf, Optical Coherence and Quantum Optics, University Press, Cambridge, 1995, p. 871.

[18] D.L. Andrews, P. Allcock, Optical Harmonics in Molecular Systems, Wiley-VCH, Weinheim, 2002.

[19] H. Kuzmany, Solid-state Spectroscopy: An Introduction, Springer-Verlag, Berlin, 2002, p. 184

[20] G.H. Wagnière, Linear and Nonlinear Optical Properties of Molecules, Weinheim, VCH-Verlag, 1993.

[21] D.L. Andrews, D.S. Bradshaw, Eur. J. Phys. 30 (2009) 239. 